\title{
Process Mining in Supply the Need for Rule-Based Knowledge Abstraction
}

\author{
P.Shirisha
}

\begin{abstract}
Organizations struggle to achieve insight in however their business processes square measure conducted actually. Method mining allows organizations to extract this data\} by analyzing business events recorded in their information systems. However, the business events recorded in these systems don't forever mirror constant level of abstraction because the desired method model that's employed by the business. Current method mining approaches offer light attention to the current gap. This paper proposes many information preparation strategies that apply provision domain information for method mining the fabric movements among a corporation additionally, associate degree custom-made method mining project methodology is bestowed that expressly includes these preparation strategies.
\end{abstract}

Keywords - method mining, event mapping, abstraction levels, logistics, business rules.

\section{I.INTRODUCTION}

The high dependency on info systems in business processes these days has created a scenario within which the digital and also the physical world square measure well aligned. This alignment has created it potential to store giant amounts of knowledge on the activities that square measure occurring in business processes, known as event information .whereas the data systems are getting a lot of and a lot of tangled with the operational processes they support, organizations struggle with extracting information from these systems and their event information. Existing information analysis techniques and tools employed in business performance observance specialize in easy performance indicators and neglect the causative and dynamic dependencies that exist in processes. Method mining may be a comparatively new technique that fills the gap between \{data mining data methoding\} at the one hand and business process analysis and modeling on the opposite. It aims to extract process-related information from event information and allows the organization to get, monitor and improve their processes.

Process mining has become a "hot topic" in Business method Management analysis and lots of organizations have an interest in applying it the explanations for this growing interest square measure the massive quantity of method information that's being generated by info systems employed in business methods and also the incontrovertible fact that ancient data processing techniques don't seem to be capable of analyzing this information during a process central manner. The exaggerated stress in legislation on company governance additionally forces organizations to watch their business activities a lot of closely. Moreover, organizations

Revised Manuscript Received on 14 August, 2019.

P.SHIRISHA, Malla Reddy Engineering College For Women-UGC Autonomous shirishasree19@gmail.com square measure below constant pressure to enhance the performance and potency of their business processes.

When examination the events recorded during an data system to the events in a method model, organizations typically face a distinction in level of abstraction. info systems sometimes record events at a finer level of graininess than the method steps as outlined by the business users . This distinction in graininess will cause misunderstanding of method mining results because the discovered method model is a smaller amount apprehensible for the business user. To bridge the distinction in level of graininess the recorded system events ought to be mapped to apprehensible business events. This mapping effort needs sure domain information whereas current method mining project methodologies acknowledge that event information typically need preparation and improvement, they are doing not specifically address the appliance of domain information within the information preparation part.

Although loads of analysis has been conducted on the event of method mining algorithms and techniques, solely many real-life cases are studied to demonstrate their pertinency and additional worth. analysis on these real-life cases has been conducted in many domains like tending, public services and auditing. However, the appliance of method mining in supplying has not been studied extensively. This analysis so contributes to the analysis field on method mining by demonstrating its application during a complicated, giant and real-life atmosphere.

The aim of this study is to get and visualize the particular flow of materials among an outsized, international producing company. As for any producing company, managing the inventory is crucial for its structure performance. However, the corporate at that this analysis befell classified a comparatively high proportion of the inventory as inefficient. Finding the supply of this unskillfulness was a tough since the trail of the materials through the organization was typically unknown. The dimensions and quality of the corporate junction rectifier to a scattered read on the flow of materials, within which every department checked out their own piece of the puzzle. Moreover, ancient information analysis techniques couldn't analyze the fabric movement information such the way that they provided insight within the path of every individual material. thus the analysis question of this study: "how will method mining be applied to check the flow of materials".

A style science analysis approach is taken to develop many information transformation and improvement strategies that apply provision domain information. The pertinency of the designed strategies is 


\section{Process mining in supply The need for rule-based knowledge abstraction}

incontestable by a method mining project that was conducted at an outsized international company, leading to the variation of associate degree existing method mining project methodology in order that it expressly includes these information preparation steps.

\section{METHOD MINING}

In principle, tangible processes, sort of a mechanical system during a manufactory, will simply be monitored manually to assess wherever bottlenecks or failures occur. However, most business methods square measure implicit and also the information on however they're dead actually is usually unfold among the staff concerned within the process. it's true that a lot of and a lot of, business methods square measure supported these days by associate degree data system that's supported associate degree expressly sculpturesque process. However, such method models represent a simplified and idealized reality. Info on however the method is performing arts actually cannot be gained by learning such models. The necessity for organizations to achieve insight in however their business methods square measure conducted actually is one among the most important drivers behind the developments of process mining techniques. Through a mix of applied mathematics, mathematical and machine learning techniques, method mining allows organizations to: discover a method model because it is conducted actually, check the agreement of method models to reality or to boost method models with recently gained insights. Method mining will so be characterized as a mix of knowledge mining techniques and business method modeling.

The data that's used for this analysis is named a happening $\log$. Event $\log$ s contain information that's hold on by the data system on the business events or activities that befell within the business method, and carries with it 3 base elements: a case, activity and timestamp. The case, or instance of the method, is that the object that's being handled by a method (e.g. an acquisition order). The activity is that the operation that's being performed on the case. A case in a during a|in associate degree exceedingly|in a very\} business method usually starts with a call for participation of either an external or internal client (e.g. "receive client order"). The timestamp represents the time of the activity execution. Next to those base components, a happening log will carries with it further info like the resource (e.g. person or system) that performed the activity or the quantity of an acquisition order. Method mining isn't restricted to analyzing business processes solely. Any data system or machine that produces event logs, i.e. records its behavior, is analyzed victimization method mining techniques.

Since the event log contains information relating to the method execution because it is recorded within the data system implicitly additionally contains business rules and domain information. A business rule may be a logical constraint that implements a business policy and to that processes should adhere (e.g. associate degree invoice is usually checked by two employees). The term domain information is employed for rules and relations that hold during a specific atmosphere. These rules square measure typically not expressly represented however solely illustrious by domain consultants. Previous analysis has been targeted on account business rules from event logs by victimization information and/or method mining techniques, or on applying domain information to analyze/append the method model ensuing from the method mining formula. This analysis differs from the previous approaches since domain information and business rules square measure applied throughout the preparation of the information, not on the ensuing method model. Moreover, the main focus of this paper is on applying domain information and business rules, not on extracting them from event information

\section{ANALYSIS APPROACH}

This analysis is thought to be style science analysis since it aims to make associate degreed judge associate degree IT physical object - a method mining application - supposed to unravel an known structure downside. The most style downside is divided into 2 sub -problems that had to be addressed so as to review the impact of method mining within the context of supplying. the primary nested downside was the appliance of method mining techniques, that the analysis question "how to use method mining?" must be answered, i.e. what steps square measure to be taken. For this nested downside, the planning physical object may be a (revised) method mining methodology (Section IV). The second nested downside is that the execution of those steps within the supplying domain, i.e. what strategies are accustomed performs the mandatory methodology steps. the planning physical object of this sub step consists of one or two of knowledge abstraction tools (Section V). The results of those sub-problems were then accustomed answer the most analysis question (Section VI).

\section{REVISED METHOD MINING PROJECT METHODOLOGY}

In order to handle the primary sub-problem, the method Mining Project Methodology (PM2) by van Eck et al. (2015) was used as a tenet for applying method mining in follow. However, it had been found that many information preparation steps that were vital throughout this study weren't expressly mentioned during this methodology. Thus, a revised method mining project methodology was created that expressly enclosed these steps. Fig. 1, depicts the revised method mining project methodology that has the designed information preparation strategies. so as to confirm the generalizability, and so pertinence, of the revised methodology the information preparation steps square measure classified as necessary or nonobligatory. Necessary steps square measure the extraction of event information and, assumptive that the extracted information isn't perfect, the improvement of knowledge. nonobligatory information preparation steps would like solely to be performed once the context of the project needs it.

\section{A. Project formatting}

The first part of the revised method mining project methodology consists of 2 steps: shaping the scope and goal, and also the designing of the project. Input that's required for this part square measure structure queries and processes. The output of this part is one or a lot of analysis queries, the scope of those analysis queries and a project designing. In our case 
study, the initial goal of the project was to use method mining to get the flow of materials of a selected method: the fabric quality examination process. Once the goal of discovering the particular flow of materials was complete, a replacement iteration of the project was conducted to analysis if the discovered actual flow of materials complied to the assumed flow of materials by the business. The insights from the second iteration junction rectifier to a 3rd iteration of the project to assess the impact of sure flow deviations.

\section{B. Information Preparation}

The second part of the revised method mining project methodology consists of many information preparation steps. Input that's required for this part consists of the analysis question, the relevant data system and business information. The output of this part may be a dataset that's appropriate for method mining, i.e. a happening log. within the original there's one information preparation step (called information processing) mentioning many activities: making views, aggregating events, enriching $\operatorname{logs}$ and filtering logs. However, this study found that these and a lot of activities were essential for the success of the project - in line with what has been reportable on several alternative method mining comes in follow. Thus, the PM2 methodology was custom-made to specifically embrace one or two of knowledge preparation steps. Moreover, these steps address the challenges relating to the appliance of method mining as known by Bose and IEEE Task Force on method Mining on processing. creating the information preparation steps specific additionally has 2 vital advantages: it will increase the transparency of \{the method|the method\} mining process, and thus responsibility, of its result; and it stimulates the encryption of the process steps in rule-based reusable and auditable scripts.

- Data extraction: within the information extraction task \{the data|the info|the info\} that's needed to answer the analysis question is extracted from the relevant information system\&\#40;s\&\#41; to see what information is needed many queries ought to be answered, such as:

1. "What graininess is required for the analysis" o "Which amount of the method is to be

analyzed?

2. "Which information attributes square measure required for the analysis?"

3. "Which correlation between the information is used for the extraction?"

An additional question is additional within the revised PM2 methodology, replacement the information the info the information 3 process activity "creating views" that within the original PM2 befell when data extraction,

"What ought to be used as a novel symbol for a method instance?"

- Data merging: in relative databases, an immediate link between information tables required for the analysis doesn't forever exist. Merging these tables will need further tables. Considering the distinctive symbol within the information extraction task is so vital as a result of this can additionally determine further link tables that square measure required to extract or construct a novel symbol.

- Data improvement: information cleaning is a very important step to confirm correctness of the method mining results, i.e. to confirm that the dataset properly reflects the activities that occurred actually. A distinction is created during this methodology between improvement information and checking information. Improvement information consists of deleting records, or values, that square measure deemed orthogonal, incorrect, noise or outliers.

- Data checking: whereas information improvement deletes unwanted values, information checking identifies records with incorrect or missing events. This step so imposes business and domain logic on the dataset. Criteria to spot such incorrect records are derived from associate degree existing or discovered method model, i.e. business rules.

- Data splitting: so as to stop misunderstanding of the method mining results, it's vital that when checking the information it's split into 2 datasets: one containing correct and one containing incorrect cases.

- Data enrichment: event information is enriched with external or computed information attributes in order that further method views are created or to cut back the quality of the method mining results.

- Data transformation: the event information recorded by associate degree data system is usually not within the correct format to check a method model because the project goal(s) needs it. Reason for this is often that event information is hold on object.

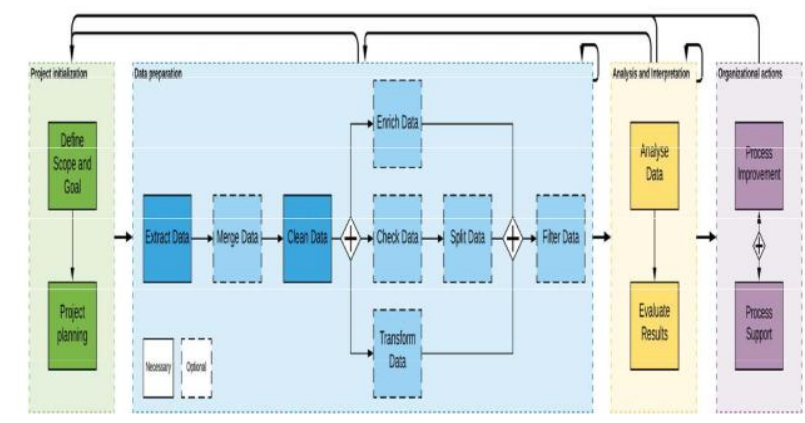

centric rather than method centrical in associate data system. Therefore, remodeling the information into a distinct format may well be required. The first PM a pair of methodology consisted of a knowledge process activity "Aggregating events" during which events square measure incorporate or divided. Complicated information transformation supported business rules and/or domain logic isn't mentioned.

- Data filtering: so as to specialize in a particular (sub) process, the event information may be filtered on sure attributes. Whereas filtering may be worn out most method mining package tools, filtering the information before the analysis can improve the method mining formula performance. Moreover, filtering improves the maintainability of the information preparation section since one larger dataset may be processed before dividing it into smaller subsets.

\section{C.Analysis and Interpretation}




\section{Process mining in supply The need for rule-based knowledge abstraction}

Once the information preparation section is complete, the information may be analyzed with method mining techniques. it's vital to involve business specialists within the analysis of the results as they will give further context to validate the findings and to confirm that the results square measure taken properly. Inputs for this section are: the event $\log$ from the information preparation section, the analysis queries from the project data format section and, if offered, existing method models for correspondence checking. The output of this section is method information within the variety of answers to the analysis queries and different findings.

\section{D.Structure Actions}

The final section of the revised method mining project methodology is that the structure actions section. During this section, the findings from the previous phases function input for creating structure changes and enhancements. The implementation of those enhancements is commonly a separate project because it needs a distinct experience and methodology, e.g. Lean or Six alphabetic character methodologies. Method mining techniques may be wont to monitor the effectiveness of the enforced changes or to support organizations in their daily operations by police work deviating running cases, predicting case behavior or advocate case actions to perform

\section{V.INFORMATION PREPARTATION STRATEGIES}

A. Extracting Material Movement information

The company at that this analysis came about uses the SAP ERP to support their internal logistical and business processes. By respondent the information extraction queries, as mentioned previous section, four tables were known that contained data associated with material movements. Fig. 2, depicts these tables and their relationship. The MSEG and MKPF tables contained information regarding material movements inside the organization. The SER03 table served as a link table between the MSEG and OBJK table, that contained the distinctive combination of fabric variety and serial variety, the distinctive case symbol during this analysis. These four tables were extracted and incorporate, leading to one dataset containing four. 2 million records that painted each material movement at one company location within the year a pair of016.

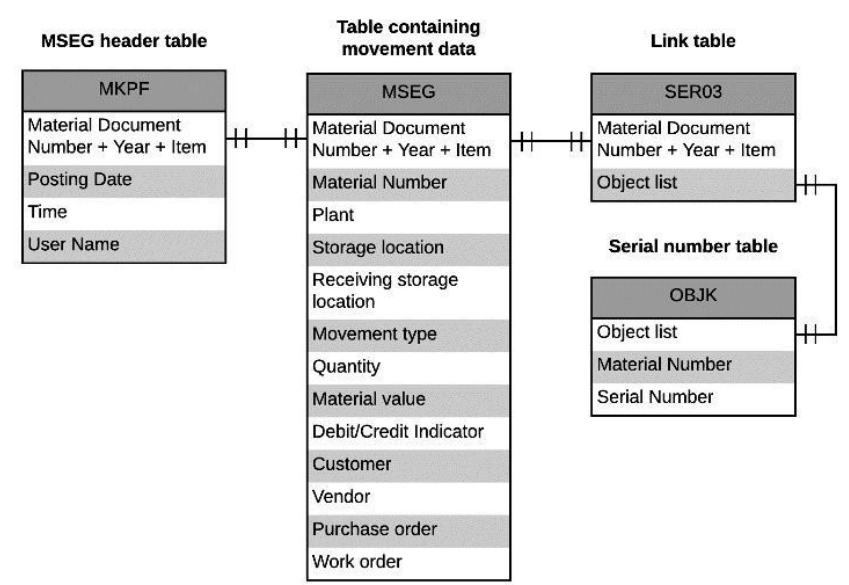

B. Removing unnecessary records
An initial analysis of the info, mistreatment the mix of SAP location and movement sort because the activities within the method, semiconductor diode to the insight that the ensuing dataset contained records that were supererogatory for the goal of visualizing the flow of materials. the explanation for this can be that once a fabric movement is entered within the system, 2 records square measure created: one that removes material from the stock at the recent location and one that adds the fabric to the stock at the new location. Consequently, this logic ends up in 2 material movements to be shown within the method mining results whereas essentially just one movement befell. Thus, for visualizing the flow of materials solely the records with a positive (debit) quantity were deemed relevant as they indicated future material location. The records with a negative (credit) quantity, i.e. removing the fabric at the present location, were deleted.

Next to it, the initial analysis semiconductor diode to the insight that the interpretation of the 2 columns representing the causing and receiving storage location ought to be understood otherwise in keeping with the record quantity. If the number is negative, the columns ought to be understood in keeping with their name, i.e. they represent the present location the fabric is aloof from and therefore the new storage location that's receiving the fabric. Once the number is positive but, this interpretation reverses, as portrayed within the log example in table one. The proper interpretation of those columns was particularly vital within the resultant checking and transformation ways.

\section{Information Enrichment}

Another finding of the initial information analysis was that the fabric movement information was overlarge and sophisticated to envision the flow of materials. The fabric movement information was characterized by high heterogeneousness, as there have been 346 doable movement sorts, and 12.702 doable storage location outlined in SAP, leading to Associate in Nursing astronomical variety of doable combos of movement sort and placement. The initial information Associate in Nursingalysis thus resulted in an incomprehensible "spaghetti" model. So as to cut back the complexness of the method mining results the dataset was enriched with four extra fields:

- Locations operate: the function, or kind of activity that occurred at the situation relating to materials (e.g. warehouse, analysis location etcetera). This enrichment befell by making a mapping table that contained every location occurring within the dataset and its corresponding operate. These functions were provided by the business knowledgeable or accountable workers.

- Movements operate \& reverse indication mapping: the function a movement sort represents: send to-, receive at, or send \& receive (transfer posting). a further column was other that indicated if the movement sort was a reverse booking that rolled back the previous material movement. Again, this enrichment befell by making a mapping table containing each movement sort that occurred within the dataset with its corresponding operate. These functions were created by consulting workers and SAP documentation.

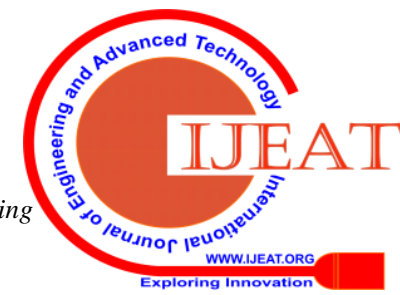


- Process relevant mapping: so as to quickly filter the info for specific sub-processes, a mapping table was created that indicated the relevant locations during a bound method. This was done by consulting the process's stakeholders, WHO indicated that SAP locations were utilized in their method. This way, the info preparation section might be conducted on the complete material movement dataset that contained many methods that might be filtered out mistreatment the other process relevant column.

- Artificial locations: not all locations a fabric might be affected to were outlined as storage locations in SAP (e.g. keep at external party for repair). This resulted in null values within the storage location columns. However, since every movement sort indicates a particular movement logic, the situation outside the scope of SAP might be derived. Again, a mapping table was created, containing the movement sort and artificial location it indicated.

\section{D.Information Checking and rending}

Once the info was clean and enriched a second iteration of the analysis section semiconductor diode to the insight that the dataset contained incorrect records, in terms of method logic. These records created strange material flows that were known by the business specialists throughout the analysis of the method mining results. These flows were deemed strange since they portrayed Associate in Nursing incorrect and unknown sequence of fabric movements. the explanation for these strange flows was either that the sequence of movement functions was incorrect (e.g. a causing record wasn't followed by a receiving record) or the sequence of locations were incorrect (e.g. send to A, received at B). 3 doable causes were found for these incorrect sequences: initial, the fabric movement might are entered in SAP while not a serial variety, leading to the record to be missing from the dataset because the distinctive id was supported the serial variety. Second, the fabric movement might are entered with the incorrect serial variety, leading to the fabric movement to be shown within the wrong case. Third, the fabric movement wasn't entered in SAP. so as to assess the case integrity, these incorrect or missing records required to be known. 2 algorithms were created that marked such records within the dataset.

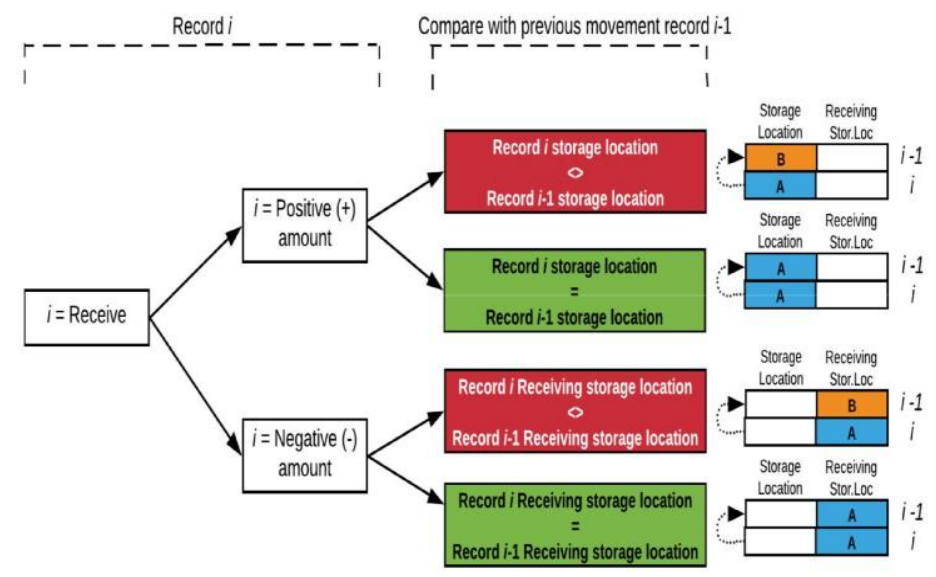

E. information Transformation
Next to the cleansing, enrichment and checking of the info, it had been found that the quality material movement info in SAP didn't end in the specified method visualization. Method mining the quality dataset resulted during a visualization that portrayed the movement of materials from a work flow perspective, i.e. the sequence of movement varieties and locations. Knowledgeable to envision the compliance of the work flow, it didn't offer a holistic read on the flow of materials between locations. Moreover, as a result of the mixture of movement kind and placement was accustomed represent the activities, many distinctive instances of a similar location were created. Fig. 4, depicts this work flow visualization within the bottom row, during which for instance location B is portrayed doubly. Thus, to check the flow of materials between locations, the info required to be remodeled into a format that might end in a visualization of the flow of materials between distinctive locations. the highest row in Fig. 4, depicts this info, the center row depicts the ensuing location flow perspective. The yellow dotted location indicates that the placement is a man-made location, which means that it's not expressly keep in SAP as a location however was derived from the movement kind.

To transform the info into the specified location perspective format Associate in Nursing algorithmic rule was created that combined 2 consecutive material movement records into one location record, supported business logic relating to material movements and also the enriched movement perform column. The mixture of receive and send records were combined into one record containing the beginning (received) and finish (send) timestamp of a location. the mixture of send and receive records weren't combined since they represent the time a fabric is in transfer. However, some exceptions existed during which the send and receive records diagrammatic the time a fabric was at a man-made location, e.g. once a fabric was send to Associate in Nursing external location. These artificial locations were once more known supported the movement kind (e.g. 541 indicate that the fabric was send to the vendor).

Since the transformation algorithmic rule was supported the previous outlined movement perform logic, it had been necessary that the dataset was checked beforehand on cases containing movement sequence errors. While not these previous checking and cacophonous steps taken, the transformation algorithmic rule couldn't have worked properly and would have created a location record that didn't represent reality. Thus, applying business and domain information in information cleansing, checking and transformation strategies was necessary to use method mining in apply. Given their importance, and also the undeniable fact that these strategies weren't expressly mentioned within the PM2 by van Eck et al. (2015), the revised method mining methodology was created. 


\section{Process mining in supply The need for rule-based knowledge abstraction}

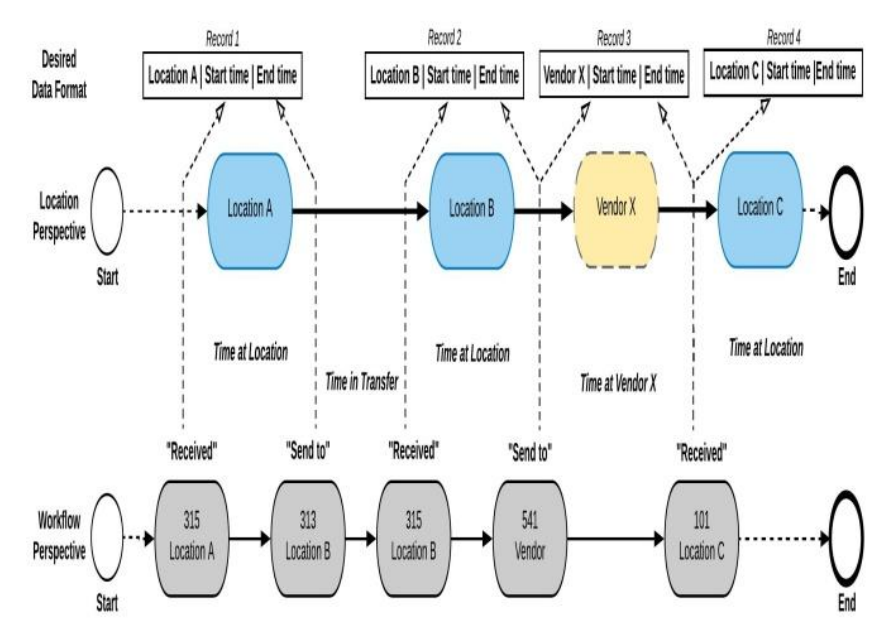

VI. RESULTS

Once the 2 sub-design issues were addressed, leading to a dataset that envisioned the specified material flow between locations, the impact of method mining in supply can be studied. For this purpose, elect|a particular\} method was selected of that a business professional indicated that it had been a possible supply of inefficient inventory. During this method, defective materials were repaired or the standard of materials that were deemed unfit for production was checked. The potential supply of inventory inefficiencies was caused by the actual fact that the materials keep at these locations weren't employed in the weekly assets designing (MRP) calculation. Thus, materials staying too long during this method were in danger of being unnecessary reordered whereas they were being repaired or requalified. Moreover, very little transparency existed within the actual performance of the method, like insight within the actual outturn time of the materials. Current performance measurements were targeted on single locations and were calculated supported the amount materials acquiring and out. The preceding path of the materials through the organization before they were keep at a location was unknown. By summary the typical time pay at every individual location, associate approximation of the method outturn time was calculated. Moreover, the outturn time was calculated supported a likely set of locations that the method consisted of. Deviating locations weren't taken into thought, creating the calculated outturn time not solely inaccurate however additionally biased. The goal of applying method mining during this method was so twofold:

1) Identify inefficiencies and their impact on the performance of the method

2) Create additional transparency within the actual performance of the method.

As a primary step within the analysis the created location log was filtered so it consisted solely of cases that contained a minimum of one scrutiny method relevant location record. \{The method \} mining tool Celonis was wont to analyze this dataset and build a process mental image. The discovered material flows of this scrutiny method square measure shown in Fig. 5. Note that the flows shown in Fig. 5, square measure the foremost common flows, low frequency occurring deviations aren't shown. Whereas the flow of materials within the method was designed, and assumed, to be linear, the method mining results showed that in point of fact this wasn't the case. Moreover, many locations that were antecedently unknown to be concerned within the method were discovered. This mental image therefore achieved the goal of making additional transparency within the actual performance of the method. It provides associate unbiased read on what locations square measure concerned within the method, and the way materials flow between them. Moreover, it gave insight within the path individual materials followed through the organization, facultative associate correct calculation of the particular outturn time of the materials within the method. What is more, it gave insight within the compliance of the method, in terms of the right order of locations that was antecedently impracticable.

Next to increasing the transparency of the method, the compliance check achieved the goal of characteristic inefficiencies and their impact on the performance of the method. Fig. 6, shows associate example of a method deviation that was antecedently unknown. the present performance measurements and corresponding key performance indicators (KPI's) were targeted on single locations. This resulted in adverse behavior of causation materials back and forth between locations (in Fig. 6, between the Analysis Location and Analysis Done Location) to stay the time pay at every location low. in point of fact but, the outturn time of those materials was up to 3 times more than the materials following the right order of locations. Thus, method mining enabled the organization to spot and assess the impact of inefficiencies on the performance of the organization.

The insights gained by applying method mining to ascertain the fabric flows light-emitting diode to 2 recommendations. Initial of all, since method mining provided a holistic read on the flow of materials throughout the organization, the present method model relating to the sequence of locations ought to be tailored so it accurately reflects reality. Second, the present location targeted performance livements ought to get replaced for method central KPI's that properly measure the behavior and performance of the method, since method mining techniques

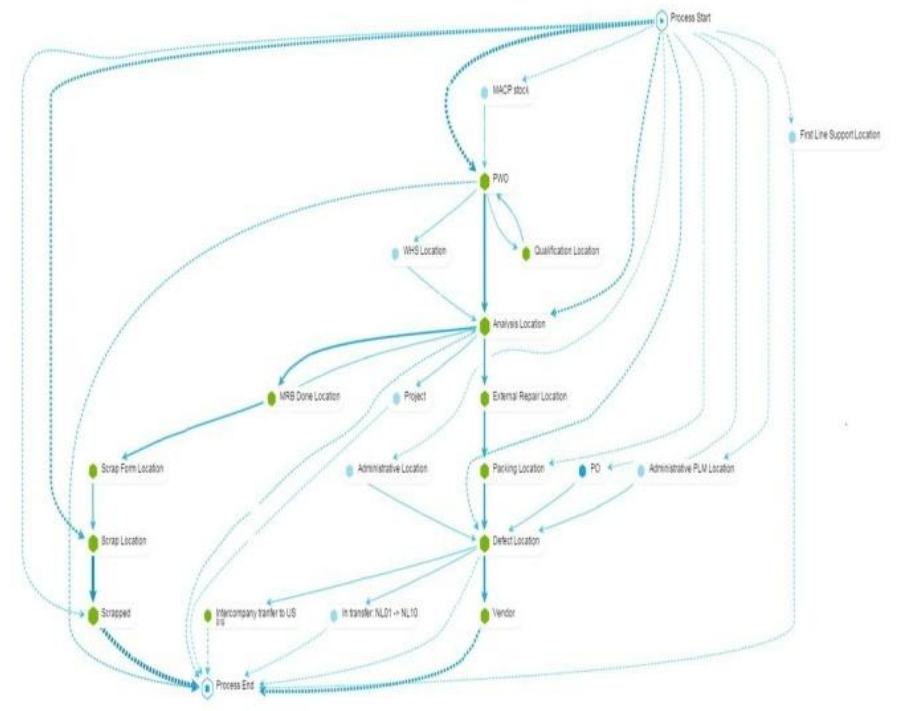

Blue Eyes Intelligence Engineering \& Sciences Publication 
Enable the organization to calculate the particular outturn time of every individual material.

\section{DISCUSSIONS AND LIMITATIONS}

The findings of this analysis don't seem to be the results of one effort to examine the flow of materials. Many iterations of the information preparation section and analysis section were conducted to succeed in the required conclusion. However, more internal validation of the results is needed. The ways and results bestowed during this analysis ar supported the idea that the domain data provided by the business skilled is valid and complete. Thus, the applied domain data should be valid to mitigate the danger that it's restricted or imperfect. for instance this, in AN earlier iteration of the Analysis section an unskillfulness just like the one represented in Fig. 6, was discovered. However, when consulting the managers to blame for the locations that were concerned within the "back and forth" causation of materials, it absolutely was found that the functions of those locations were incorrect, leading to strange behavior to be shown within the method mining results. Thus, AN adjustment to the placement functions and a brand new analysis iteration was needed to confirm an accurate material flow mental image. This illustrates that applying method mining in observe needs A repetitious approach during which there's shut collaboration between the business consultants and information analysts. particularly once domain data is applied, collaboration and validation by the business is crucial for the right interpretation of the method mining results. One ought to detain mind but that endeavor the accountable staff to validate (and so acknowledge) method deviations poses the danger of adverse behavior and will impact the structure commitment to applying method mining.

While this analysis with success applied method mining in observe, leading to many designed information preparation ways and a revised method mining project methodology, many limitations ar recognized. Initial of all, the generalizability and pertinence of the information preparation ways ar supported the actual fact that they're designed in keeping with the business logic in SAP ERP. However, this could even be seen as a limitation of the analysis as they're supported the domain logic captured during a single system. However, the actual fact that the ERP of SAP could be a advanced system that's employed by various organizations with world material flows makes that the logic of those ways within reason general.

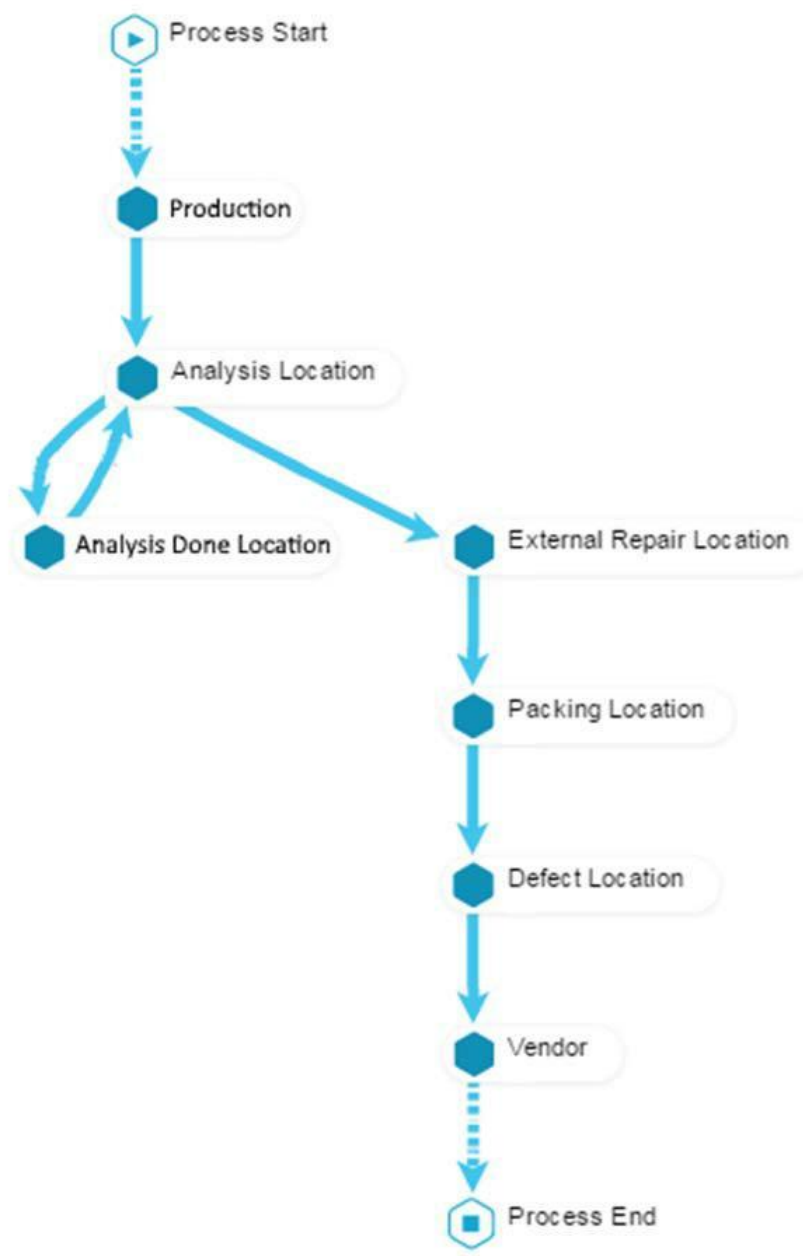

Second, the revisions created to the PM2 by van Eck et al. (2015) ar supported the actual fact that the appliance of domain data was needed during this analysis. this might not be the case for a lot of generic business processes. To accommodate for the ex gratia application of domain and business logic in method mining, many steps within the information preparation section were classified as ex gratia. more analysis ought to be conducted to validate if this classification holds true for business processes yet, or that the appliance of business and domain logic is usually needed in some kind. Method from an information perspective, i.e. the case integrity, it conjointly ensures that the information that's employed in the method mining analysis really reflects reality. This step was necessary during this analysis since the information was remodeled to the next level of abstraction. once method mining is applied to examine a work flow or business method, the logic of that method is inherently checked. more analysis ought to be conducted to search out out if information checking is just necessary once the information is remodeled to higher levels of abstraction or that it should be performed. Still, a crucial conclusion that we have a tendency to draw is that once information is remodeled to the next level, this abstraction step ought to be supported business rules, and therefore the integrity of the information (at every level) in terms of compliance to the business rules ought to be addressed. Or in other words, compliance as a method mining goal ought to be seen as a multi-level idea, wherever method compliance at the best level is just one concern. 


\section{Process mining in supply The need for rule-based knowledge abstraction}

\section{CONCLUSION}

This analysis has addressed the analysis gap on applying method mining in reality cases, by conducting method mining at an oversized and complicated transnational company. Not solely is that the another price of method mining within the field of provision incontestible, however it's conjointly been found that applying method mining in observe isn't a trivial task. A revised method mining project methodology is bestowed that takes into thought that the business events recorded in AN system ar of a special level of graininess than business users need, so requiring a a lot of elaborate information preparation section. the requirement for information abstraction has been expressed earlier.These papers recommend algorithms like agglomeration to urge at purposeful activities and sub-activities from event information. Our approach is completely different in 2 ways: (a) by wishing on domain data and (b) by specializing in abstractions that correspond to business rules (integrity rules), instead of simply co-occurrence. However, we have a tendency to don't exclude a mix of our approach with the formal information abstraction mechanisms developed within the different work. The latter will be wont to recommend abstraction rules that may be upraised to integrity rules on the idea of domain data.

It is incontestable that so as to use method mining to examine the flow of materials, information transformation is required that takes provision business logic into thought. a change technique is bestowed that addresses the distinction in event graininess for the fabric movements in SAP, along side 2 information checking ways that make sure that the information represents material movements that occurred really. By applying the designed information preparation ways in observe, the another price of the designed artifacts is incontestable. Moreover, it shows that material movement information will be analyzed to supply 2 views on the flow of materials: a work flow perspective and a location perspective. The mental image of the flow of materials from a location perspective provided helpful insights on the performance and compliance of the fabric flows. Deviating flows and their impact on the entire outturn time were known. Next to Last, the information checking technique was found to be crucial to confirm correct method mining results. Not solely will this checking technique offer a lot of insight within the compliance of the, it absolutely was found that the performance of locations differed per flow variant, i.e. the sequence of locations. Applying method mining so enabled AN unbiased and a lot of correct analysis of the performance and compliance of the flow of materials and individual locations inside that flow.

Two recommendations were created supported insights gained by method mining. First, the prevailing method model ought to be adjusted in order that it reflects reality a lot of correct. Second, new method centrical KPIought to be created that live the performance of the flow of materials a lot of accurately.

Our analysis suggests that compliance within the context of method mining ought to be seen as a multi-level idea. The formalization of this idea could be a topic for future analysis. we have a tendency to expect it to be not solely essential within the provision domain, however conjointly for several auditing applications; this can be another analysis topic.

\section{REFERENCES}

1. Baier, T., Mendling, J., Weske, M. (2014) Bridging abstraction layers in process mining. Information Systems, 46, 123-139. http://dx.doi.org/10.1016/j.is.2014.04.004

2. Bose, R. P. J. C., Mans, R. S., \& van der Aalst, W. M. P. (2013). Wanna Improve Process Mining Results ? It's High Time We Consider Data Quality Issues Seriously. In IEEE Symposium on Computational Intelligence and Data

3. Mining (CIDM 2013) (pp. 127-134) https://doi.org/10.1109/CIDM.2013.6597227

4. De Weerdt, J., Schupp, A., Vanderloock, A., \& Baesens, B. (2013) Process Mining for the multi-faceted analysis of business processes - A case study in a financial services organization. Computers in Industry, 64(1), 57-67. https://doi.org/10.1016/j.compind.2012.09.010

5. Dixit, P. M., Buijs, J. C. A. M., van der Aalst, W. M. P., Hompes, B. F. A., Buurman, J. (2017). Using domain knowledge to enhance process mining results. In Data-Driven Process Discovery and Analysis: 5th IFIP WG 2.6 International Symposium, SIMPDA 2015, Vienna, Austria, December 9-11, 2015, Revised Selected Papers (pp. 76-104). (Lecture Notes in Business Information Processing;

6. Vol. 244). Dordrecht:

https://doi.org/10.1007/978-3-319-53435-0_4

Springer.

7. Günther, C. W. (2009). Process Mining in Flexible Environments. In Lecture Notes in Computer Science (pp.

8. 1-360). Retrieved http://www.springerlink.com/index/9YH5WYAWLWV20 UAE.pdf

9. IEEE Task Force on Process Mining. (2012). Process mining manifesto. Business Process Management Workshop 2011 (Vol. 99) Springer-Verlag. https://doi.org/10.1007/978-3-642-28108-2_19

10. Tiwari, A., Turner, C. J., \& Majeed, B. (2008). A review of business process mining: State-of-the-art and future trends. Business Process Management Journal, 14(1), 5-22. https://doi.org/10.1108/14637150810849373

11. van der Aalst, W.M.P., Reijers, H. A., \& Song, M. (2005). Discovering social networks from event logs. Computer Supported Cooperative Work, 14, 549-593. https://doi.org/10.1007/s10606-005-9005-9

12. van der Aalst, W. M. P., Reijers, H. A., Weijters, A. J. M. M., van Dongen, B. F., Alves de Medeiros, A. K., Song, M., \& Verbeek, H. M. W. (2007). Business process mining: An industrial application. Information Systems, 32, 713-732. https://doi.org/10.1016/j.is.2006.05.003

13. van der Aalst, W.M.P. (2011). Process Mining: Discovery,

14. Conformance and Enhancement of Business Processes.

15. Berlin https://doi.org/10.1007/978-3-642-19345-3

16. van der Aalst, W. (2013). "Mine your own business": using process mining to turn big data into real value. In ECIS 2013 Completed Research (Vol. 225, pp. 1-9). Retrieved from http://aisel.aisnet.org/ecis2013_cr

17. van Eck, M. L., Lu, X., Leemans, S. J. . J., \& van der Aalst, W. M. P. (2015). PM2: A process mining project methodology. In International Conference on Advanced Information Systems Engineering (pp. 297-313).

18. https://doi.org/10.1007/978-3-319-19069-3_19

19. Wieringa, R. J. (2014). Design Science Methodology for Information Systems and Software Engineering. Berlin

20. Heidelberg: Springer-Verlag. https://doi.org/10.1145/1810295.1810446

21. Smirnov, S., Reijers, H. \& Weske, M. (2012) From fine-grained to abstract process models: A semantic approach, Information Systems, 37 (8), pp. 784-797, https://doi.org/10.1016/j.is.2012.05.007.

22. Tax, N., Sidorova, N., Haakma, R., van der Aalst, W.M.P. (2016) Event abstraction for process mining using supervised learning techniques. In Proceedings of the SAI Intelligent Systems Conference, pp. 161-170. Springer.

23. Becker, T., \& Intoyoad, W. (2017). Context Aware Process Mining in Logistics. Procedia CIRP, 63, 557-562. 